\title{
Disparities in health care determine prognosis in newly diagnosed glioblastoma
}

\author{
Ankush Chandra, MS, Jonathan W. Rick, BS, Cecilia Dalle Ore, BS, Darryl Lau, MD, \\ Alan T. Nguyen, BS, Diego Carrera, MD, Alexander Bonte, BA, Annette M. Molinaro, PhD, \\ Philip V. Theodosopoulos, MD, Michael W. McDermott, MD, Mitchel S. Berger, MD, and \\ Manish K. Aghi, MD, PhD
}

Department of Neurological Surgery and Brain Tumor Research Center, University of California, San Francisco, California

OBJECTIVE Glioblastoma (GBM) is an aggressive brain malignancy with a short overall patient survival, yet there remains significant heterogeneity in outcomes. Although access to health care has previously been linked to impact on prognosis in several malignancies, this question remains incompletely answered in GBM.

METHODS This study was a retrospective analysis of 354 newly diagnosed patients with GBM who underwent first resection at the authors' institution (2007-2015).

RESULTS Of the 354 patients (median age 61 years, and $37.6 \%$ were females), $32(9.0 \%)$ had no insurance, whereas $322(91.0 \%)$ had insurance, of whom $131(40.7 \%)$ had Medicare, $45(14 \%)$ had Medicaid, and $146(45.3 \%)$ had private insurance. On average, insured patients survived almost 2-fold longer $(p<0.0001)$ than those who were uninsured, whereas differences between specific insurance types did not influence survival. The adjusted hazard ratio (HR) for death was higher in uninsured patients (HR 2.27 [95\% Cl 1.49-3.33], $p=0.0003$ ). Age, mean household income, tumor size at diagnosis, and extent of resection did not differ between insured and uninsured patients, but there was a disparity in primary care physician (PCP) status - none of the uninsured patients had PCPs, whereas $72 \%$ of insured patients had PCPs. Postoperative adjuvant treatment rates with temozolomide (TMZ) and radiation therapy (XRT) were significantly less in uninsured (TMZ in 56.3\%, XRT in 56.3\%) than in insured (TMZ in 75.2\%, XRT in 79.2\%; $p=0.02$ and $p=$ 0.003 ) patients. Insured patients receiving both agents had better prognosis than uninsured patients receiving the same treatment (9.1 vs 16.34 months; $p=0.025$ ), suggesting that the survival effect in insured patients could only partly be explained by higher treatment rates. Moreover, having a PCP increased survival among the insured cohort (10.7 vs 16.1 months, HR $1.65[95 \% \mathrm{Cl} 1.27-2.15] ; p=0.0001$ ), which could be explained by significant differences in tumor diameter at initial diagnosis between patients with and without PCPs ( 4.3 vs $4.8 \mathrm{~cm}, p=0.003)$, and a higher rate of clinical trial enrollment, suggesting a critical role of PCPs for a timelier diagnosis of GBM and proactive cancer care management.

CONCLUSIONS Access to health care is a strong determinant of prognosis in newly diagnosed patients with GBM. Any type of insurance coverage and having a PCP improved prognosis in this patient cohort. Higher rates of treatment with TMZ plus XRT, clinical trial enrollment, fewer comorbidities, and early diagnosis may explain survival disparities. Lack of health insurance or a PCP are major challenges within the health care system, which, if improved upon, could favorably impact the prognosis of patients with GBM.

https://thejns.org/doi/abs/10.3171/2018.3.FOCUS1852

KEYWORDS glioblastoma; insurance; prognosis; primary care physician; PCP; socioeconomic; health care

$\mathrm{G}$ LIOBLASTOMA (GBM) is the most common primary adult brain tumor, with a median survival of less than 2 years and a 5 -year survival rate of less than $5 \% .^{13,21}$ In spite of advancements in medicine and a better understanding of GBM tumor biology, there remains significant heterogeneity in patient survival. Thus, it is imperative to explore modifiable factors at the patient level to provide the optimal care to overcome disparities in outcomes. In spite of efforts to expand health care access, large disparities in treatments and outcomes remain among our

ABBREVIATIONS $\mathrm{CCI}=$ Charlson Comorbidity Index; $\mathrm{GBM}=$ glioblastoma; $\mathrm{HR}=$ hazard ratio; $\mathrm{PCP}=$ primary care physician; $\mathrm{SEER}=$ Surveillance, Epidemiology, and End Results; $\mathrm{TMZ}$ = temozolomide; $\mathrm{XRT}=$ radiation therapy.

SUBMITTED February 2, 2018. ACCEPTED March 29, 2018.

INCLUDE WHEN CITING DOI: 10.3171/2018.3.FOCUS1852. 
populations and affect the prognosis of cancer patients. ${ }^{31,35}$ In a population-based study by Walker et al., the authors revealed that patients with one of the 10 most deadly cancers (excluding all brain malignancies) had worse survival outcomes if they were uninsured or had Medicaid coverage ${ }^{35}$ Moreover, studies by Curry et al. and Momin et al. demonstrated that in patients with craniotomies, insurance status affected prognosis, with privately insured patients enjoying a better overall survival when compared to those with Medicaid or those who were uninsured. ${ }^{4,18}$

Although several socioeconomic factors have been shown to contribute to disparities in health care, ${ }^{20,22,23}$, ${ }^{29,31}$ insurance status and primary care physician (PCP) status have not been studied as extensively in the context of survival in patients with GBM at a single-institution level. To date, 2 studies have found an association between insurance status and prognosis in patients with GBM; however, these studies were based on the Surveillance, Epidemiology, and End Results (SEER) Program database and the National Cancer Database and could not define patient-based factors that could explain disparities in outcomes. ${ }^{22,26}$ However, no studies to date have explored the impact of PCP status on GBM prognosis.

We sought to address this knowledge gap by analyzing the impact of insurance status as well as PCP status on median survival in adult patients ( $>18$ years old) with newly diagnosed GBM at a single, tertiary, specialized brain tumor center. We also sought to define various other factors associated with survival in this patient cohort. Here we report the survival benefits of insurance and PCP status as well as propose mechanisms for improved survival; specifically, higher rates of treatment with temozolomide (TMZ) and radiation therapy (XRT) as well as lower comorbidities at diagnosis in insured patients. Also, among insured patients with PCPs, we report the impact of these PCPs on timely diagnosis of the disease and enrollment in the latest clinical trials.

\section{Methods \\ Cohort Selection}

We performed a retrospective analysis of data from patients with newly diagnosed GBM undergoing their first resection between 2007 and 2015 at our tertiary care specialized brain tumor center. Any patient under the age of 18 years, patients who had been previously diagnosed with a lower-grade glioma, who previously had undergone resection and/or received adjuvant therapy, or who had an unknown insurance status were excluded from our study. In total, 354 patients met our inclusion criteria. Our institutional committee on human research reviewed and approved this study. Because our study did not use information beyond medical records, informed consent of patients was not required.

\section{Recorded Parameters}

Age at the time of surgery was defined as the patient's age on the date of his/her first resection. Tumor size was defined as its maximum diameter on the patient's preoperative MRI. Extent of resection was divided into 3 groups based on radiological reports: the first group consisted of patients in whom gross-total resection of enhancing tumor was achieved, the second group included patients in whom subtotal resection of enhancing tumor was achieved, and the third group consisted of patients who underwent only a biopsy. Length of stay was defined as the time between date of admission and discharge as per medical records. Survival (in months) was calculated by determining the time between date of first resection and date of death (found in medical records or through the Social Security Death Index).

All postoperative treatments after first resection were recorded, including the standard of care Stupp protocol, ${ }^{33}$ concomitant XRT and adjuvant TMZ, and any "agents in addition to Stupp protocol" after first resection or at recurrence. Other patient-specific factors recorded included ethnicity, sex, insurance type (uninsured, Medicare, Medicaid, and private insurance), PCP status, and rates of comorbidities compiled through chart review of patient electronic records. Comorbidities were analyzed by 2 methods: first, by using the Charlson Comorbidity Index (CCI), which assigns a weighted score to patient comorbidities based on severity; ${ }^{3}$ and second, by reporting rates of preoperative patient comorbidities by pooling and strictly including type II diabetes mellitus, hyperlipidemia, hypertension, cardiovascular diseases, neurological disorders, other malignancies, and pulmonary, hepatic, and renal diseases. The median household income associated with the patient's zip code of primary residence was recorded, as per the US census website (http://factfinder.census.gov/ servlet/ACSSAFFFacts).

\section{Statistical Analysis}

The chi-square test and Student t-test were used for categorical and continuous variable comparison, respectively. For any number $<5$, the Fisher exact test was performed for categorical variables. Kaplan-Meier analyses with log-rank tests were used for univariate survival analysis. Multivariate Cox proportional hazard analysis was used for multivariate survival analysis and to calculate hazard ratios (HRs) with 95\% CIs. The threshold for statistical significance for all tests was $\mathrm{p}<0.05$, and all probability values were 2-tailed. All analyses were performed using JMP Statistical Software version 12 (SAS Institute Inc.).

\section{Results}

\section{Patient Cohort}

During our study period, data from the electronic medical records of 354 patients who met our inclusion criteria were analyzed. The median age of our cohort was 61 years, and $37.6 \%(n=133)$ were female, with the majority of our cohort being Caucasian $(n=275,77.7 \%)$ (Table 1). Analysis of socioeconomic status revealed that the majority of our cohort was insured, with only $9 \%(\mathrm{n}=32)$ patients uninsured. Among the 322 insured patients, $40.7 \%$ $(\mathrm{n}=131)$ were covered under Medicare, $14 \%(\mathrm{n}=45)$ under Medicaid, and $45.3 \%(\mathrm{n}=146)$ had private insurance coverage. The majority of our cohort $(n=232,65.5 \%)$ had an assigned PCP, whereas 122 patients (34.5\%) did not have an assigned PCP. 


\section{Comparisons Between Subgroups Stratified by Insurance Status}

Next, we compared patients and their characteristics with respect to insurance status, grouped into no insurance and any insurance coverage (Table 2). Patients without insurance tended to be younger than those who were insured (59 vs 62 years); however, this difference was not significant $(p=0.2)$. The rate for having a PCP was significantly higher in all the insured subgroups when compared to the uninsured cohort $(\mathrm{p}<0.001)$, with at least half of the patients in each of the groups-Medicare $(n=101,77.1 \%)$, Medicaid $(n=23,51.11 \%)$, and private insurance $(n=108$, $74 \%$ )-having PCP coverage, whereas none of the uninsured had PCP coverage (Table 3). Tumor size (diameter) was larger in uninsured patients compared to every one of the insured subgroups except Medicaid patients (who had the largest tumor size); however, this finding was not significant $(\mathrm{p}=0.3)$. Preoperative patient comorbidities were significantly more prevalent in the uninsured group compared to the insured group $(75 \%$ vs $51.55 \%, \mathrm{p}=0.01)$, although there was no significant difference between CCI scores.

\section{Effect of Insurance Status on Postoperative GBM Treatments Implemented}

As expected, we found that the rates of postoperative TMZ were significantly lower in uninsured patients as compared to the insured cohort $(\mathrm{p}=0.02)$, with just over half of the uninsured patients receiving TMZ ( $\mathrm{n}=$ $18,56.3 \%$ ), whereas about three-fourths of the insured cohort received TMZ $(n=242,75.2 \%)$. A similar trend was observed with XRT, wherein the uninsured cohort had a significantly lower rate of treatment $(n=18,56.3 \%$; $\mathrm{p}=0.003)$ in comparison to the insured group $(\mathrm{n}=255$, $79.2 \%$ ). When comparing rates of clinical trial enrollment and rates of treatment with agents in addition to the Stupp protocol, there was no significant difference between the insured and uninsured cohorts (Table 2). However, on subgroup analysis, the rates of these treatments varied significantly (Table 3 ) between the uninsured, Medicare, Medicaid, and private insurance group, with privately insured patients having the highest rates of clinical trial enrollment $(36.3 \%, \mathrm{n}=53 ; \mathrm{p}=0.003)$ and treatment with additional agents $(55.5 \%, \mathrm{n}=81 ; \mathrm{p}=0.0004)$.

\section{Effect of Insurance Status on GBM Outcomes}

The uninsured cohort had a significantly lower rate of postoperative treatment with XRT $(\mathrm{n}=18,56.3 \%$; $\mathrm{p}$ $=0.0005)$ and TMZ $(\mathrm{n}=18,56.3 \% ; \mathrm{p}=0.0005)$ than the Medicare $(\mathrm{n}=95,72.5 \% ; \mathrm{n}=86,65.7 \%)$, Medicaid $(\mathrm{n}=$ $30,66.7 \% ; n=29,64.4 \%)$, and private insurance $(n=124$, $84.9 \% ; \mathrm{n}=121,82.9 \%$ ) groups.

The median survival of all our patients was 13.9 months. When assessing survival among the 4 groups, uninsured patients had a significantly lower overall survival (8.8 months) when compared to the Medicare (15.4 months), Medicaid (14.5 months), and private insurance (18.6 months) subgroups ( $\mathrm{p}<0.001)$. Using Kaplan-Meier univariate survival analysis (Fig. 1), patients who had any type of insurance coverage survived almost twice as long
TABLE 1. Demographics and socioeconomic status

\begin{tabular}{cc}
\hline \multicolumn{1}{c}{ Variable } & Value \\
\hline No. of patients & 354 \\
\hline Sex (no., \%) & $133(37.57)$ \\
\hline Female & $221(62.43)$ \\
\hline Male & $61,18-88$ \\
\hline Median age in yrs, range & \\
\hline Ethnicity (no., \%) & $275(77.69)$ \\
\hline Caucasian & $58(16.38)$ \\
\hline Non-Caucasian & $21(5.93)$ \\
\hline Unknown & $32(9.04)$ \\
\hline Insurance type & $322(90.96)$ \\
\hline Uninsured & $131(40.68)$ \\
\hline Insured & $45(13.98)$ \\
\hline Medicare & $146(45.34)$ \\
\hline Medicaid & \\
\hline Private & $232(65.54)$ \\
\hline PCP (no., \%) & $122(34.46)$ \\
\hline Yes & \\
\hline No & $45,370.50$ \\
\hline Median household income $(\$)$ & $50,054.50$ \\
\hline No insurance & $39,863.00$ \\
\hline Medicare & \\
\hline Medicaid & \\
\hline Private insurance & \\
\hline
\end{tabular}

as those who did not have insurance coverage (8.8 vs 15.2 months; HR 2.27 [95\% CI 1.49-3.33], p < 0.0001). Moreover, survival analysis based on comorbidities revealed that the insured patient cohort experienced almost twice as long a survival when compared to the uninsured cohort (7.4 vs 13.2 months; HR 1.94 [95\% CI 1.17-3.07], $\mathrm{p}=$ 0.007 ), suggesting that higher rates of comorbidities play a role in worse survival in uninsured patients.

Survival analysis by PCP status within the insured patient cohort (Fig. 2) revealed that patients with an assigned PCP also had a significantly longer survival (by approximately $60 \%$; 16.1 months) compared to those without a PCP (10.7 months; HR 1.65 [95\% CI 1.27-2.15] p = $0.0001)$. When looking for potential factors that could explain the survival benefit in insured patients, we found that patients with PCPs tended to have a smaller tumor burden at diagnosis when compared to those without PCPs (4.3 vs $4.8 \mathrm{~cm}, \mathrm{p}=0.003$; see Table 5) as well as a higher enrollment in clinical trials postoperatively $(18.9 \%$ vs $29.7 \%$, p $=0.04)$, suggesting the role of PCPs in timely diagnosis of the disease and timely enrollment in the latest clinical trials. Moreover, patients with no PCP had significantly lower rates of postoperative treatment with XRT (67.8\% vs $83.6 \%, \mathrm{p}=0.003)$ and TMZ (62.2\% vs $80.2 \%, \mathrm{p}=0.0008)$ when compared to those with PCPs. Survival analysis by PCP status of patients enrolled in clinical trials revealed a significantly longer survival period (18.5 vs 22 months; $\mathrm{HR}=1.97$ [95\% CI 1.05-3.53], $\mathrm{p}=0.025)$, a finding consistent with the literature. ${ }^{8,9}$ 
TABLE 2. Distribution of insured and uninsured patients

\begin{tabular}{|c|c|c|c|}
\hline Variable & No Insurance & Any Insurance & $\mathrm{p}$ Value \\
\hline No. of patients & 32 & 322 & \\
\hline Sex (no. female, \%) & $6(18.75)$ & $127(39.44)$ & 0.0218 \\
\hline Median age in yrs (range) & $59(19-83)$ & $62(18-88)$ & 0.176 \\
\hline Median household income in $\$ 1000$ s (range) & $45.37(25-128.35)$ & $52.43(19.95-140.90)$ & 0.676 \\
\hline \multicolumn{4}{|l|}{ PCP status (no., \%) } \\
\hline Yes & $0(0)$ & $232(72.05)$ & \\
\hline No & $32(100)$ & $90(27.95)$ & $<0.001$ \\
\hline \multicolumn{4}{|l|}{ Tumor characteristics } \\
\hline Mean diameter in $\mathrm{cm}(95 \% \mathrm{Cl})$ & $4.56(3.96-5.26)$ & $4.44(4.26-4.62)$ & 0.72 \\
\hline \multicolumn{4}{|l|}{ Extent of resection (no., \%) } \\
\hline Gross-total & $15(46.88)$ & $115(35.71)$ & 0.40 \\
\hline Subtotal & $16(50)$ & $200(62.11)$ & \\
\hline Biopsy & $1(3.13)$ & $7(2.17)$ & \\
\hline Mean \pm SD length of stay in days & $4.30 \pm 2.09$ & $4.83 \pm 3.30$ & 0.39 \\
\hline Reop at first recurrence (no., \%) & $8(25)$ & $75(23.29)$ & 0.82 \\
\hline Comorbidities at diagnosis (no., \%) & $24(75)$ & $166(51.55)$ & 0.01 \\
\hline \multicolumn{4}{|l|}{$\mathrm{CCl}$ score (no., \%) } \\
\hline 0 & $17(53.13)$ & $218(67.7)$ & 0.23 \\
\hline 1 & $8(25)$ & $63(19.57)$ & \\
\hline$\geq 2$ & $7(21.88)$ & $41(12.73)$ & \\
\hline \multicolumn{4}{|l|}{ Postop adjuvant treatment (no., \%) } \\
\hline Radiation therapy & $18(56.25)$ & $255(79.2)$ & 0.003 \\
\hline TMZ & $18(56.25)$ & $242(75.16)$ & 0.02 \\
\hline Clinical trials & $9(28.13)$ & $86(26.71)$ & 0.86 \\
\hline Agents in addition to Stupp protocol & $13(40.63)$ & $138(42.86)$ & 0.81 \\
\hline \multicolumn{4}{|l|}{ Median postop survival in mos $(95 \% \mathrm{Cl})$} \\
\hline Overall & $8.82(5.55-10.59)$ & $15.22(13.61-16.64)$ & $<0.0001$ \\
\hline XRT plus TMZ & $9.14(5.29-16.04)$ & $16.34(14.9-18.21)$ & 0.025 \\
\hline Patients with comorbidities & $7.35(2.73-13.15)$ & $13.18(11.28-15.22)$ & 0.007 \\
\hline
\end{tabular}

Boldface type indicates statistical significance.

In a Cox proportional hazard model, multivariate analysis to identify independent predictors of survival in all patients in our study $(\mathrm{n}=354)$ revealed that an older age at surgery (> 65 years; HR 1.36 [95\% CI 1.03-1.76], $\mathrm{p}=0.003$ ), having "no insurance" (HR 1.81 [95\% CI $1.15-2.72, \mathrm{p}=0.01$ ), and a CCI score $\geq 1$ (HR 1.49 [95\% CI $1.03-2.12], \mathrm{p}=0.03$ ) were independent predictors of shorter survival (Table 4), which is consistent with the literature. ${ }^{10,15,19,26}$ In the same multivariate model, treatment with both TMZ and XRT (HR 0.60 [95\% CI 0.45-0.81, p $=0.001)$ and postoperative clinical trial enrollment (HR 0.54 [95\% CI 0.41-0.71, p < 0.0001) were independent predictors of improved overall survival in our patient cohort.

\section{Discussion}

Although health care disparities have been shown to have a global impact on disease progression and prognosis in different malignancies, access to care has not been studied as extensively in GBM. ${ }^{31}$ Thus, we sought to characterize the factors affecting health care access and their im- pact on prognosis of patients with newly diagnosed GBM. Consistent with the literature, our cohort comprised 9.04\% uninsured patients, $12.7 \%$ with Medicaid, $37.01 \%$ with Medicare, and $41.24 \%$ with private insurance. ${ }^{22,26}$ In this single-institution study, we found insurance status, one of the many indicators of access to health care, to be a major prognostic factor for patients with newly diagnosed GBM, consistent with the findings in a recent study by Rong et al. ${ }^{26}$ in which a large population cancer registry was used.

Previous studies have shown improved outcomes in cancer patients who had insurance coverage..$^{25,32,35}$ In a study by Aizer et al., insured patients had a decreased likelihood of metastatic presentation as well as a decreased rate of death in 11 different cancer indications. ${ }^{1}$ Rosenberg et al. revealed that, in adolescent and young adult populations with 8 different cancer types, lacking insurance coverage was independently related to a higher stage of malignancy and a greater risk of death, irrespective of the stage of the disease. ${ }^{27}$ As mentioned earlier, to date only 2 studies have looked at the impact of insurance status on survival in adult patients with GBM. In a population- 
TABLE 3. Presentation of patients under different insurance types

\begin{tabular}{|c|c|c|c|c|c|}
\hline Variable & No Insurance & Medicare & Medicaid & Private & $\mathrm{p}$ Value \\
\hline No. of patients & 32 & 131 & 45 & 146 & \\
\hline Sex (no. female, \%) & $6(18.75)$ & $48(36.64)$ & $19(42.22)$ & $60(41.1)$ & 0.212 \\
\hline Median age in yrs (range) & $59(19-83)$ & $69(45-88)$ & $58(21-81)$ & $55(18-77)$ & $<0.0001$ \\
\hline Median household income in $\$ 1000$ s (range) & $45.37(25-128.35)$ & $50.05(19.95-137.19)$ & $39.86(20.5-92.38)$ & $59.72(20.78-140.90)$ & $<0.0001$ \\
\hline PCP status (no., \%) & & & & & 0.001 \\
\hline Yes & $0(0)$ & $101(77.1)$ & $23(51.11)$ & $108(73.97)$ & \\
\hline No & $32(100)$ & $30(22.9)$ & $22(48.89)$ & $38(26.03)$ & \\
\hline \multicolumn{6}{|l|}{ Tumor characteristics } \\
\hline Mean diameter in $\mathrm{cm}(95 \% \mathrm{Cl})$ & $4.56(3.96-5.26)$ & $4.25(3.97-4.53)$ & $4.76(4.27-5.26)$ & $4.53(4.27-4.80)$ & 0.274 \\
\hline \multicolumn{6}{|l|}{ Extent of resection (no., \%) } \\
\hline Gross-total & $15(46.88)$ & $43(32.82)$ & $12(26.67)$ & $60(41.10)$ & 0.34 \\
\hline Subtotal & $16(50)$ & $86(65.65)$ & $31(68.89)$ & $83(56.85)$ & \\
\hline Biopsy & $1(3.13)$ & $2(1.53)$ & $2(4.44)$ & $3(2.05)$ & \\
\hline Mean \pm SD length of stay in days & $4.30 \pm 2.09$ & $4.9 \pm 3.59$ & $6.55 \pm 4.12$ & $4.21 \pm 2.50$ & $<0.001$ \\
\hline Reop at first recurrence (no., \%) & $8(25)$ & $19(14.5)$ & $10(22.22)$ & $46(31.51)$ & 0.011 \\
\hline Comorbidities at diagnosis (no.,\%) & $24(75)$ & $88(67.18)$ & $19(42.22)$ & $59(40.41)$ & $<0.0001$ \\
\hline \multicolumn{6}{|l|}{ CCl score (no., \%) } \\
\hline 0 & $17(53.13)$ & $76(58.02)$ & $29(64.44)$ & $113(77.4)$ & 0.0002 \\
\hline 1 & $8(25)$ & $25(19.08)$ & $13(28.89)$ & $25(17.12)$ & \\
\hline$\geq 2$ & $7(21.88)$ & $30(22.90)$ & $3(6.67)$ & $8(5.48)$ & \\
\hline Median survival in mos $(95 \% \mathrm{Cl})$ & $8.82(5.55-10.59)$ & $15.35(12.13-17.85)$ & $14.53(9.6-25.01)$ & $18.64(16.34-21.40)$ & $<0.001$ \\
\hline \multicolumn{6}{|l|}{ Postop adjuvant treatment (no., \%) } \\
\hline Radiation therapy & $18(56.25)$ & $95(72.52)$ & $30(66.67)$ & $124(84.93)$ & 0.0005 \\
\hline TMZ & $18(56.25)$ & $86(65.65)$ & $29(64.44)$ & $121(82.88)$ & 0.0005 \\
\hline Clinical trials & $9(28.13)$ & $28(21.37)$ & $5(11.11)$ & $53(36.30)$ & 0.003 \\
\hline Agents in addition to Stupp protocol & $13(40.63)$ & $40(30.53)$ & $17(37.78)$ & $81(55.48)$ & 0.0004 \\
\hline
\end{tabular}

Boldface type indicates statistical significance.

based study by Rong et al., using the SEER database, the authors found that patients with non-Medicaid insurance had a longer median overall survival, whereas those with Medicaid or no insurance did not have this survival benefit. ${ }^{26}$ In our study we found similar results to that of Rong et al., with our insured patient cohort having a median overall survival that was almost twice as long when compared to survival of uninsured patients. However, unlike the findings by Rong et al., in our study there was no statistical significance in the median overall survival among the Medicaid, Medicare, and private insurance groups, which could potentially reflect greater equality between these coverages in our regional market compared to the diverse states that comprise the SEER database.

Despite worse survival in the uninsured cohort, we did not find that tumor size differed between the insured versus noninsured patients, findings that are contrary to those well documented in the literature. ${ }^{4,26}$ Tumor size is just one marker of disease burden, and our retrospective study did not fully capture other metrics such as Karnofsky Performance Scale score. However, the impact of access to health care on neurological diseases and survival outcomes has been supported by several other studies, corroborating our findings. In a Nationwide Inpatient Sample study by Curry et al., 99,665 craniotomies performed between 1998 and
2004 were analyzed, in which the authors showed that uninsured patients or those covered by Medicaid had higher mortality rates when compared to patients who had private insurance. Additionally, the authors found that patients who had limited access to health care presented with a more severe disease. ${ }^{4}$ Similarly, Shin et al. analyzed 4325 cases of anaplastic astrocytoma and found that patients covered by private insurance have a significantly longer overall survival than those covered by Medicaid or Medicare, or who are uninsured. ${ }^{28}$ In another study using the National Inpatient Sample data on 28,581 craniotomies for brain tumors, Momin et al. found that patients who were uninsured had almost twice the mortality rate of those who had private insurance $(2.6 \%$ vs $1.3 \%, \mathrm{p}<0.001)$. When the authors adjusted for patient characteristics, uninsured patients still posed a higher risk of experiencing in-hospital death when compared to those who were privately insured (HR 2.62; 95\% CI 1.11-6.14; $\mathrm{p}=0.03) .{ }^{18}$

Besides worse survival, we found that our uninsured cohort was undertreated with XRT and TMZ and lacked access to a PCP. Only a little over half of our uninsured cohort received TMZ and XRT, which was significantly lower than in the insured patient group. However, insured patients undergoing therapy with both TMZ and XRT survived almost 7.2 months longer than uninsured patients 


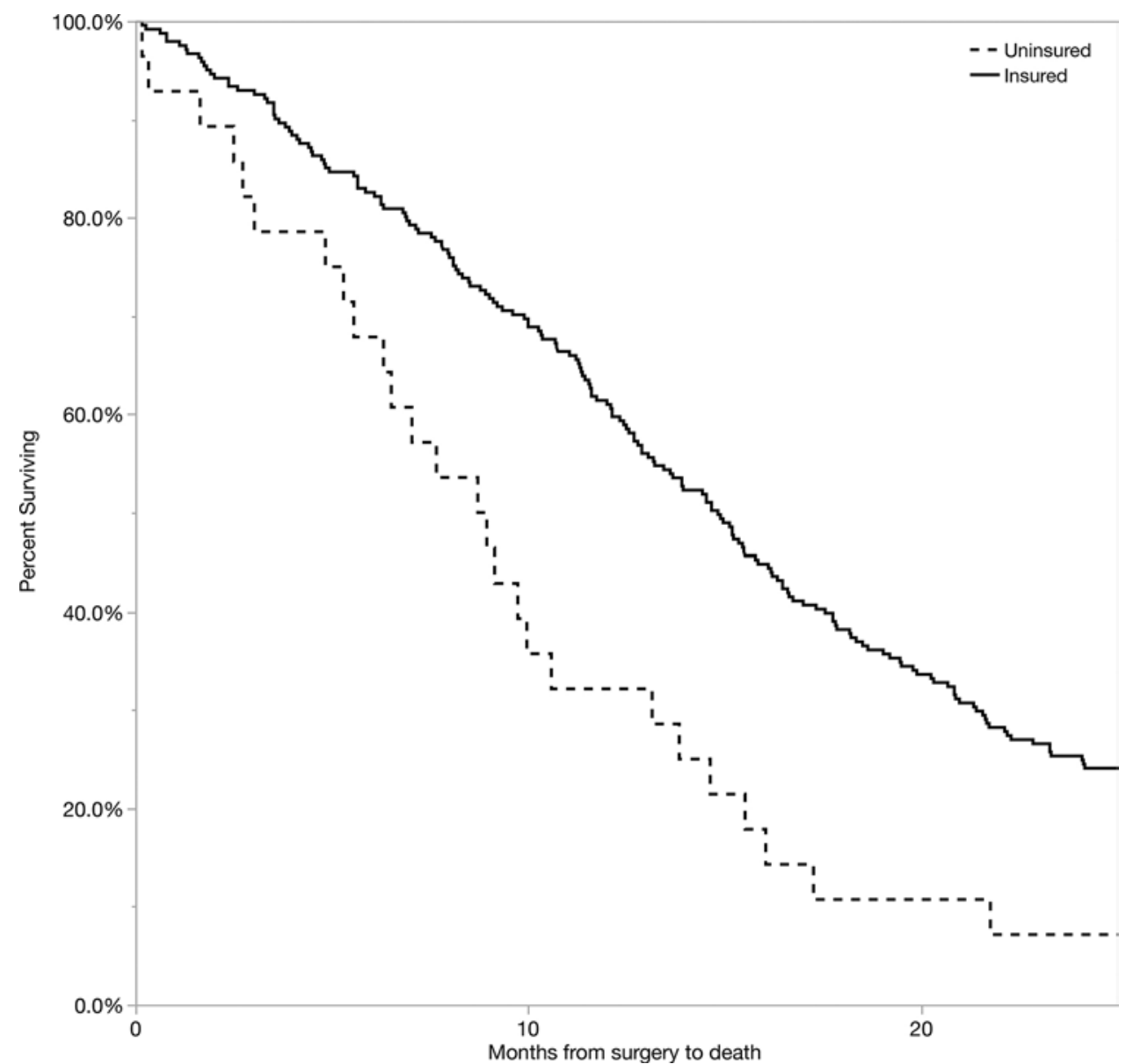

FIG. 1. Kaplan-Meier analysis by insurance status in patients with GBM. Time until death in patients with insurance (solid line) and no insurance (dashed line) $(p<0.0001)$.

receiving these treatments $(p=0.03)$, suggesting that failure to receive chemotherapy and radiation was just one explanation for the poor survival of uninsured patients in our cohort. In a recent study by Pollom et al., the authors found that there was a delay in receiving XRT in patients with GBM who were uninsured or on Medicaid, and these patients did not enjoy the survival benefit seen in the cohort receiving XRT within 35 days of GBM resection. ${ }^{22}$ In 2005, Stupp et al. found that postresection XRT and adjuvant TMZ led to a significant increase in overall survival in GBM, and this protocol has thus since become the gold standard for GBM patients. 33

Although the Stupp protocol improved survival in patients with primary GBM significantly, in a study by Wasserfallen et al., the authors reported that TMZ concurrently used with XRT for maintenance treatment of GBM increased direct costs of treatment 8-fold when compared to the pre-TMZ period. Moreover, the authors determined that the cost per quality-adjusted life year of the Stupp protocol ranged from $\$ 55,731.88$ to $\$ 72,251 .{ }^{36}$ The cost of this treatment is likely to make it challenging for uninsured patients to self-pay for treatment or find charitable funding sources, and may even contribute to the reduced survival seen in patients with Medicaid or Medicare coverage relative to private insurance in the SEER study of Rong et al., ${ }^{26}$ a difference not found in our study. Although the CCI, a validated scale for analyzing patient comorbidities, did not reveal a significant difference in comorbidities between insured and uninsured patients, we did find higher rates of comorbidities in uninsured than insured patients. This difference offers another explanation for worse survival in uninsured patients, given that studies have shown that comorbidities such as obesity and hyperlipidemia, ${ }^{11,30}$ among others, that are not captured by the CCI affect survival in patients with GBM.

Another interesting finding, which we report for the first time, is the impact of PCP status on the survival of patients with GBM. In our insured cohort $72.1 \%(n=232)$ of patients had a designated PCP, whereas none of the uninsured patients had PCP coverage ( $n=0, p<0.001)$. Moreover, we found that having a designated PCP increased survival by approximately $50 \%$ in our patient cohort (10.34 vs 15.85 months; HR 1.71 [95\% CI 1.32-2.19]; $\mathrm{p}<0.0001$ ) as well as increased survival by about $60 \%$ when comparing insured patients by PCP status (10.72 vs 16.11 months; HR 1.65 [95\% CI 1.27-2.15]; $\mathrm{p}=0.0001$ ) (Table 5). A number of studies have reported and emphasized the importance of a PCP's role in screening, early diagnosis, and management of several malignancies. ., $, 12,17,34$ Moreover, PCPs play a major role in cancer survival, and their engagement with patients allows them to provide regular and improved follow-up care. ${ }^{16}$ 


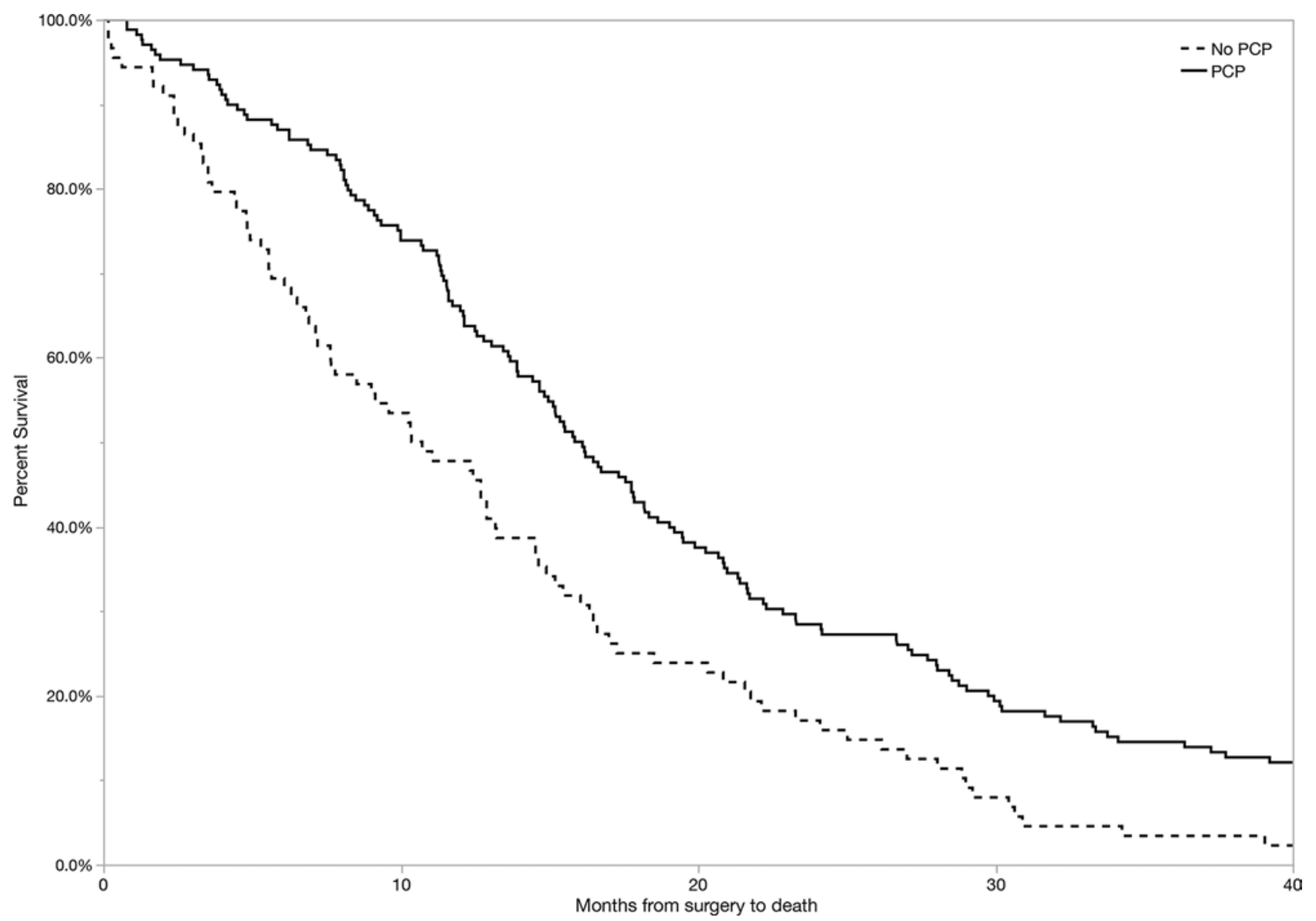

FIG. 2. Kaplan-Meier analysis by PCP status in insured patients with GBM. Time until death in patients with designated PCP (solid line) and no PCP (dashed line) $(p=0.0001)$.

A recent study by Easley et al. defined the roles of family physicians in cancer care, which include coordinating cancer care, managing patient comorbidities, and providing psychosocial care to patients. ${ }^{6}$ Thus, it is not surprising that patients with a designated PCP have better survival outcomes when compared to their counterparts. When looking at possible variables that could explain the disparity in survival within the insured cohort, we found that patients who did not have PCPs had a larger tumor burden at diagnosis when compared to those with PCP coverage, supporting the notion of the role of PCPs in early diagnosis of disease.? Another likely explanation for this disparity in survival could be different patterns of treatment for patients with and without PCPs, because PCPs have shown to play a critical role in managing routine cancer care. ${ }^{6,89}$ This is evident in our study in which we found that within the insured cohort, patients without PCPs had significantly lower rates of treatment with TMZ and XRT, and lower enrollment in clinical trials, which is reflected in their overall survival (Table 5).

\section{Limitations of the Study}

As a retrospective analysis, our study is subject to the usual limitations. These limitations included limited availability of patient data in electronic medical records. For instance, in some patients the insurance status was un- available in their charts, and thus we had to exclude them, which led to a smaller than desired sample size, limiting the strength of our study. Also, several patient charts were missing the Karnofsky Performance Scale score and cause of death, and thus we were unable to report it in our study. Although US zip codes have been used by many studies to determine median income for patients, ${ }^{5,14,24}$ this method may not provide data that accurately portray actual patient income.

Additionally, because our institution is a tertiary specialized brain tumor center, our patients are referred from different parts of the country. In most cases we lacked many details from the medical history in patient charts,

TABLE 4. Cox proportional hazard multivariate analysis of factors associated with survival of patients with glioblastoma

\begin{tabular}{lccc}
\hline \multicolumn{1}{c}{ Factor } & HR & p Value & $95 \% \mathrm{Cl}$ \\
\hline $\begin{array}{l}\text { Older age at surgery }(>65 \text { yrs } \\
\text { old vs } \leq 65 \text { yrs old) }\end{array}$ & 1.36 & 0.003 & $1.03-1.76$ \\
\hline No insurance & 1.81 & 0.01 & $1.15-2.72$ \\
\hline Higher CCl score (0 vs $\geq 1)$ & 1.49 & 0.03 & $1.03-2.12$ \\
\hline XRT plus TMZ therapy & 0.60 & 0.001 & $0.45-0.81$ \\
\hline Postop clinical trial enrollment & 0.54 & $<0.0001$ & $0.41-0.71$ \\
\hline
\end{tabular}


TABLE 5. Subgroup distribution of patients without and with PCP within the insured cohort

\begin{tabular}{|c|c|c|c|}
\hline Variable & No PCP & PCP & p Value \\
\hline No. of patients & 90 & 232 & \\
\hline Sex (no. female, \%) & $39(43.33)$ & $91(39.22)$ & 0.63 \\
\hline Median age in yrs (range) & $61(19-88)$ & $62.5(18-85)$ & 0.40 \\
\hline Median household income in $\$ 1000$ s (range) & $47.66(20.5-128.35)$ & $55.36(19.95-140.90)$ & 0.44 \\
\hline \multicolumn{4}{|l|}{ Tumor characteristics } \\
\hline Mean diameter in $\mathrm{cm}(95 \% \mathrm{Cl})$ & $4.84(4.58-5.11)$ & $4.29(4.06-4.51)$ & 0.003 \\
\hline \multicolumn{4}{|l|}{ Extent of resection (no., \%) } \\
\hline Gross-total & $37(41.11)$ & $78(33.62)$ & 0.14 \\
\hline Subtotal & $53(58.89)$ & $147(63.36)$ & \\
\hline Biopsy & $0(0)$ & $7(3.02)$ & \\
\hline Mean \pm SD length of stay in days & $5.62 \pm 4.01$ & $4.39 \pm 2.67$ & 0.002 \\
\hline Reop at first recurrence (no., \%) & $17(18.89)$ & $58(25)$ & 0.24 \\
\hline Comorbidities at diagnosis (no., \%) & $47(52.22)$ & $129(55.60)$ & 0.13 \\
\hline \multicolumn{4}{|l|}{$\mathrm{CCl}$ score (no., \%) } \\
\hline 0 & $65(72.22)$ & $153(65.95)$ & 0.55 \\
\hline 1 & $15(16.67)$ & $48(20.69)$ & \\
\hline$\geq 2$ & $10(11.11)$ & $31(13.36)$ & \\
\hline \multicolumn{4}{|l|}{ Postop adjuvant treatment (no., \%) } \\
\hline Radiation therapy & $61(67.78)$ & $194(83.62)$ & 0.003 \\
\hline TMZ & $56(62.22)$ & $186(80.17)$ & 0.0008 \\
\hline Clinical trials & $17(18.89)$ & $69(29.74)$ & 0.048 \\
\hline Agents in addition to Stupp protocol & $33(36.67)$ & $105(45.26)$ & 0.16 \\
\hline \multicolumn{4}{|l|}{ Median postop survival in mos $(95 \% \mathrm{Cl})$} \\
\hline Overall median survival & $10.72(7.63-13.18)$ & $16.11(13.94-18.18)$ & 0.0001 \\
\hline XRT plus TMZ & $14.53(12.33-16.47)$ & $17.56(15.19-19.50)$ & 0.004 \\
\hline Enrollment in postop clinical trials & $18.51(12.89-23.28)$ & $22.01(18.35-28.44)$ & 0.025 \\
\hline
\end{tabular}

Boldface type indicates statistical significance.

especially from physician and hospital visits prior to visiting our center. Thus, we could not record and analyze the effects of frequency of PCP visits and the impact on survival in patients with GBM.

\section{Conclusions}

Our study demonstrates that access to health care, indicated by insurance status and PCP status, is an important and strong determinant of prognosis in patients with newly diagnosed GBM, in addition to age at surgery and receipt of Stupp protocol treatment. We showed for the first time that having a PCP benefits overall survival in patients with newly diagnosed GBM. Thus, work is needed to provide optimal care and treatment by expansion of health insurance and quicker access to PCPs for the patients with GBM who have the greatest disparities in treatments and outcomes. Future studies that can define mechanisms to overcome the barriers to accessing health care are further warranted.

\section{Acknowledgments}

Ankush Chandra and Jonathan Rick are fellows of Howard Hughes Medical Institute (HHMI).

\section{References}

1. Aizer AA, Falit B, Mendu ML, Chen MH, Choueiri TK, Hoffman KE, et al: Cancer-specific outcomes among young adults without health insurance. J Clin Oncol 32:2025-2030, 2014

2. Campbell RJ, Ramirez AM, Perez K, Roetzheim RG: Cervical cancer rates and the supply of primary care physicians in Florida. Fam Med 35:60-64, 2003

3. Charlson M, Szatrowski TP, Peterson J, Gold J: Validation of a combined comorbidity index. J Clin Epidemiol 47:12451251, 1994

4. Curry WT Jr, Carter BS, Barker FG II: Racial, ethnic, and socioeconomic disparities in patient outcomes after craniotomy for tumor in adult patients in the United States, 19882004. Neurosurgery 66:427-438, 2010

5. Derakhshan A, Miller J, Lubelski D, Nowacki AS, Wells BJ, Milinovich A, et al: The impact of socioeconomic status on the utilization of spinal imaging. Neurosurgery 77:746-754, 2015

6. Easley J, Miedema B, O'Brien MA, Carroll J, Manca D, Webster F, et al: The role of family physicians in cancer care: perspectives of primary and specialty care providers. Curr Oncol 24:75-80, 2017

7. Ferrante JM, Gonzalez EC, Pal N, Roetzheim RG: Effects of physician supply on early detection of breast cancer. J A m Board Fam Pract 13:408-414, 2000

8. Field KM, Drummond KJ, Yilmaz M, Tacey M, Compston 
D, Gibbs P, et al: Clinical trial participation and outcome for patients with glioblastoma: multivariate analysis from a comprehensive dataset. J Clin Neurosci 20:783-789, 2013

9. Field KM, Rosenthal MA, Yilmaz M, Tacey M, Drummond $\mathrm{K}$ : Comparison between poor and long-term survivors with glioblastoma: review of an Australian dataset. Asia Pac J Clin Oncol 10:153-161, 2014

10. Flanigan PM, Jahangiri A, Kuang R, Truong A, Choi S, Chou A, et al: Developing an algorithm for optimizing care of elderly patients with glioblastoma. Neurosurgery 82:64-75, 2018

11. Gaist D, Hallas J, Friis S, Hansen S, Sørensen HT: Statin use and survival following glioblastoma multiforme. Cancer Epidemiol 38:722-727, 2014

12. Gikas A, Triantafillidis JK: The role of primary care physicians in early diagnosis and treatment of chronic gastrointestinal diseases. Int J Gen Med 7:159-173, 2014

13. Johnson DR, O'Neill BP: Glioblastoma survival in the United States before and during the temozolomide era. J Neurooncol 107:359-364, 2012

14. Kasl RA, Brinson PR, Chambless LB: Socioeconomic status does not affect prognosis in patients with glioblastoma multiforme. Surg Neurol Int 7 (Suppl 11):S282-S290, 2016

15. Lacroix M, Abi-Said D, Fourney DR, Gokaslan ZL, Shi W, DeMonte F, et al: A multivariate analysis of 416 patients with glioblastoma multiforme: prognosis, extent of resection, and survival. J Neurosurg 95:190-198, 2001

16. Maly RC, Liu Y, Diamant AL, Thind A: The impact of primary care physicians on follow-up care of underserved breast cancer survivors. J Am Board Fam Med 26:628-636, 2013

17. Miser WF: Cancer screening in the primary care setting: the role of the primary care physician in screening for breast, cervical, colorectal, lung, ovarian, and prostate cancers. Prim Care 34:137-167, 2007

18. Momin EN, Adams H, Shinohara RT, Frangakis C, Brem H, Quiñones-Hinojosa A: Postoperative mortality after surgery for brain tumors by patient insurance status in the United States. Arch Surg 147:1017-1024, 2012

19. Morgan ER, Norman A, Laing K, Seal MD: Treatment and outcomes for glioblastoma in elderly compared with nonelderly patients: a population-based study. Curr Oncol 24:e92-e98, 2017

20. Muquit S, Parks R, Basu S: Socio-economic characteristics of patients with glioblastoma multiforme. J Neurooncol 125:325-329, 2015

21. Ostrom QT, Gittleman H, Farah P, Ondracek A, Chen Y, Wolinsky Y, et al: CBTRUS statistical report: Primary brain and central nervous system tumors diagnosed in the United States in 2006-2010. Neuro Oncol 15 (Suppl 2):ii1-ii56, 2013

22. Pollom EL, Fujimoto DK, Han SS, Harris JP, Tharin SA, Soltys SG: Newly diagnosed glioblastoma: adverse socioeconomic factors correlate with delay in radiotherapy initiation and worse overall survival. J Radiat Res 59 (Suppl 1):i11i18, 2018

23. Porter AB, Lachance DH, Johnson DR: Socioeconomic status and glioblastoma risk: a population-based analysis. Cancer Causes Control 26:179-185, 2015

24. Reames BN, Birkmeyer NJ, Dimick JB, Ghaferi AA: Socioeconomic disparities in mortality after cancer surgery: failure to rescue. JAMA Surg 149:475-481, 2014

25. Roetzheim RG, Pal N, Tennant C, Voti L, Ayanian JZ, Schwabe A, et al: Effects of health insurance and race on early detection of cancer. J Natl Cancer Inst 91:1409-1415, 1999

26. Rong X, Yang W, Garzon-Muvdi T, Caplan JM, Hui X, Lim
$M$, et al: Influence of insurance status on survival of adults with glioblastoma multiforme: A population-based study. Cancer 122:3157-3165, 2016

27. Rosenberg AR, Kroon L, Chen L, Li CI, Jones B: Insurance status and risk of cancer mortality among adolescents and young adults. Cancer 121:1279-1286, 2015

28. Shin JY, Yoon JK, Diaz AZ: Influence of insurance status and income in anaplastic astrocytoma: an analysis of 4325 patients. J Neurooncol 132:89-98, 2017

29. Sia Y, Field K, Rosenthal M, Drummond K: Socio-demographic factors and their impact on the number of resections for patients with recurrent glioblastoma. J Clin Neurosci 20:1362-1365, 2013

30. Siegel EM, Nabors LB, Thompson RC, Olson JJ, Browning $\mathrm{JE}$, Madden MH, et al: Prediagnostic body weight and survival in high grade glioma. J Neurooncol 114:79-84, 2013

31. Singh GK, Daus GP, Allender M, Ramey CT, Martin EK, Perry C, et al: Social determinants of health in the United States: addressing major health inequality trends for the nation, 1935-2016. Int J MCH AIDS 6:139-164, 2017

32. Smith JK, Ng SC, Zhou Z, Carroll JE, McDade TP, Shah SA, et al: Does increasing insurance improve outcomes for US cancer patients? J Surg Res 185:15-20, 2013

33. Stupp R, Mason WP, van den Bent MJ, Weller M, Fisher $\mathrm{B}$, Taphoorn MJ, et al: Radiotherapy plus concomitant and adjuvant temozolomide for glioblastoma. $\mathbf{N}$ Engl J Med 352:987-996, 2005

34. Triantafillidis JK, Vagianos C, Gikas A, Korontzi M, Papalois A: Screening for colorectal cancer: the role of the primary care physician. Eur J Gastroenterol Hepatol 29:e1e7, 2017

35. Walker GV, Grant SR, Guadagnolo BA, Hoffman KE, Smith $\mathrm{BD}$, Koshy M, et al: Disparities in stage at diagnosis, treatment, and survival in nonelderly adult patients with cancer according to insurance status. J Clin Oncol 32:3118-3125, 2014

36. Wasserfallen JB, Ostermann S, Leyvraz S, Stupp R: Cost of temozolomide therapy and global care for recurrent malignant gliomas followed until death. Neuro Oncol 7:189-195, 2005

\section{Disclosures}

The authors report no conflict of interest concerning the materials or methods used in this study or the findings specified in this paper.

\section{Author Contributions}

Conception and design: Aghi, Chandra. Acquisition of data: Chandra, Rick, Dalle Ore, Nguyen, Carrera, Bonte. Analysis and interpretation of data: Aghi, Chandra, Rick, Molinaro. Drafting the article: Aghi, Chandra. Critically revising the article: Aghi, Chandra, Lau, Theodosopoulos, McDermott, Berger. Reviewed submitted version of manuscript: Aghi, Chandra, Rick, Dalle Ore, Lau, Nguyen, Carrera, Bonte, Molinaro, Theodosopoulos, McDermott, Berger. Approved the final version of the manuscript on behalf of all authors: Chandra. Statistical analysis: Chandra, Molinaro. Administrative/technical/material support: Aghi. Study supervision: Aghi, Chandra.

\section{Correspondence}

Manish K. Aghi: California Center for Pituitary Disorders, University of California, San Francisco, CA. manish.aghi@ucsf.edu. 